\title{
The pH-Triggered Amphiphilic Polycarbodiimides as Nanovesicles
}

\author{
Enosha Harshani De Silva ", Bruce Novak \\ Department of Chemistry and Biochemistry, The University of Texas at Dallas, Richardson, USA \\ Email address: \\ enoshadesilva@gmail.com (E. H. De Silva), Bruce.Novak@utdallas.edu (B. Novak) \\ ${ }^{*}$ Corresponding author
}

\section{To cite this article:}

Enosha Harshani De Silva, Bruce Novak. The pH-Triggered Amphiphilic Polycarbodiimides as Nanovesicles. American Journal of Polymer Science and Technology. Vol. 7, No. 1, 2021, pp. 16-22. doi: 10.11648/j.ajpst.20210701.13

Received: February 24, 2021; Accepted: March 22, 2021; Published: March 30, 2021

\begin{abstract}
The pH-triggered polymers are a sub-class of stimuli-responsive macromolecules. These smart polymers can experience physical or chemical transition due to small $\mathrm{pH}$ responses. $\mathrm{pH}$-dependent materials gain great demand within a short period, by considering their potential applications. pH-triggered macromolecules typically possess weak basic or weak acidic functional groups. The functional groups often used include tertiary amines, pyridines, phosphate, and carboxylic acids. These stimuli-dependent materials are ideal candidates for the biomedical field. Herein, report pH-dependent chiral amphiphilic carbodiimide polymers. Helical polycarbodiimides consist of alternating imine and amine backbone, attached to different pendant groups. Substituting the pendant groups, enable to change of the properties of polycarbodiimides. Thus, we invented polar functional groups such as dimethylamine and piperazine to the side chains to enhance the solubility in an aqueous medium. Moreover, each unit of these polymers consists of hydrophobic methyl functionality and hydrophilic dimethyl and piperazine side groups. Thus, these carbodiimide polymers were self-assembled in aqueous solutions as well as buffer solutions due to the hydrophilic and hydrophobic moieties of the polymer. The micelles can be used as nanocarriers. Furthermore, the pH-dependent swelling properties were observed in these polymeric nanoparticles in different time intervals. Thus, the $\mathrm{pH}$-triggered volume change of the micelles can be used as a controlled release, targeted site drug delivery applications.
\end{abstract}

Keywords: pH-Triggered, Micelles, Controlled Release

\section{Introduction}

pH-triggered polymers consist of either weak basic or weak acidic functional groups, that donate or accept hydrogen ions as to changes in external $\mathrm{pH}$. Tertiary amine, pyridine, phosphate, sulfonate, and carboxyl are some of the ionizing pendant groups, $[1,2]$ which allow undergoing phase and morphology changes depending on the acidic and basic conditions, these polymers can behave as cations or anions. [1, 2]

The $\mathrm{pH}$-dependent acidic materials generally comprise carboxylic, phosphonic, boronic, and sulfonic acid functional groups. $[1,2]$ On the other hand, polybases consist of morpholine, piperazine, tertiary amine, pyrrolidine, pyridine, and imidazole side groups. [1,2] pH-responsive synthetically modified materials interestingly displayed biocompatibility and degradability as natural polymers. [1,2] These materials displayed different architectures like the liner, vesicles, brushes, stars, dendrimers, and micro hydrogels. [1,2]

Smart polymers can be used for drug delivery systems, where the encapsulated drugs or molecules can be released at the specific site and the proper time depending on the slight environmental changes. The structural alterations of the polymer vary from contracting or swelling to fragmentation, depending on external environment variations such as temperature, light, ultrasound, $\mathrm{pH}$, mechanical stress, and electric stress. [1-3] However, poor response time, slow versatility, chemical and physical instability of the polymers are some disadvantages of the stimuli-responsive macromolecules. [1] Thus, there is an increasing demand for the invention of novel intelligent delivery macromolecules in the fields of tissue engineering and biomedical applications. [3]

Nanoparticles are generally considered as the diameter of 1- $100 \mathrm{~nm}$ materials. [3, 4] These nanoparticles are widely used in different nanomedicine applications including, tissue 
engineering and drug delivery vehicles. [4] These nanoscale devices can be used to replace damaged tissues and organs. [4] Polymers are prominent biomaterials widely used in nanomedicine applications. [3] The properties of these materials can be tuned by altering a broad range of monomers. [4, 5] Thus, there is an extensively growing market for novel varieties of polymeric nanomaterials. [4]

Previous works have been reported for thermodynamically stable micellization of the block-copolymers in selective solutions. [6] However comparatively fewer efforts have been done to investigate the self-assembly properties of homopolymers. [6] This self assembles polymer materials are attracted interest in the fields of drug delivery, detergents, cosmetics, microcapsules, and micro-reactors. [7-10] Preparation of self-assemble homopolymers like; poly (propyl acrylic acid), hyperbranched polyphosphate, and phenyl phosphinic acid-containing amphiphilic homopolymers recently drawn great attention due to their simple synthetic route and different micelle formation mechanism. [5]

Herein we report water-soluble polycarbodiimide polymers containing methyl, dimethylamine, and piperazine pendant groups in each repeat unit (Figure 1). The aggregation behavior of these polymers in different buffer solutions and their applications as nanocarriers have been studied.

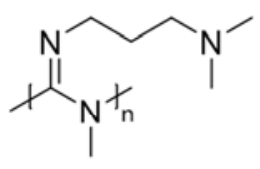

Poly-1-S

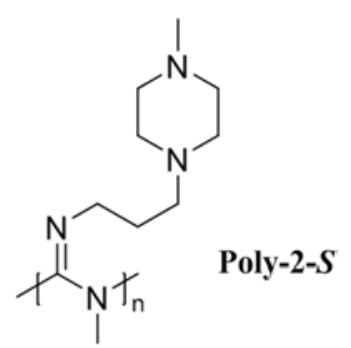

Figure 1. Proposed polymers used to study nanoparticle behavior.

\section{Materials and Experiments}

\subsection{Materials}

Analytical grade solvents and reagents are purchased form Fisher scientific and Sigma Aldrich. ( $S$ ) BINOL Ti (IV) diisopropoxide catalysts are synthesized and stored under an inert environment.

\subsection{Equipment}

The polymerization was performed in the MBraun UNILABTM glove box. Bruker AVANCE IIITM $500 \mathrm{MHz}$ NMR spectrometer used to collect NMR spectra. IR spectrums collected using Thermo Scientific Nicolet 380 ATR-FTIR spectrometer. CD (Electron Circular Dichroism) results obtained, AVIV Circular Dichroism Spectrometer $(\mathrm{c}=2.0 \mu \mathrm{mg} / \mathrm{mL}, l=50.0 \mu \mathrm{m})$, optical rotation measured, JASCO P-1010 polarimeter $(435 \mathrm{~nm}, \mathrm{c}=2.00 \mathrm{mg} / \mathrm{mL}, l=10.0$ $\mathrm{cm})$. DLS measurements were determined on Malvern Zetasizer particle sizer Nano ZS model, He-Ne (633 nm, Max $4 \mathrm{~mW}$ ) equipped as the laser source. The molecular weight of the polymer samples was analyzed using Shimadzu Prominence Modular GPC (equipped with UV-Vis detector and refractive index). Polystyrene is used as a standard and chloroform as a mobile phase. TEM analysis was conducted on high-resolution Transmission Electron Microscope -JEOL 2100 TEM (200 keV).

\subsection{Synthesis of Monomers and Polymers}

\subsubsection{3-(3-Dimethylaminopropyl)-1-methylcarbodiimide}

3-dimethylaminopropylamine $(9.21 \mathrm{ml}, 73.75 \mathrm{mmol})$ dissolved in $100 \mathrm{ml}$ of methylene chloride and methyl isothiocyanate $(5.55 \mathrm{~g}, 73.75 \mathrm{mmol})$ was gradually added into the reaction mixture at $0^{\circ} \mathrm{C}$. The reaction continued overnight and then added mercury (II) oxide $(16.00 \mathrm{~g}, 0.07 \mathrm{mmol})$ followed by calcium sulfate $(15.06 \mathrm{~g}, 0.11 \mathrm{mmol})$. Filtered out the byproducts and the remaining solution concentrated and passed through a silica column with diethyl ether as the solvent. The obtained product was yellow color liquid. Yield (54\%). ${ }^{1} \mathrm{H} \quad \mathrm{NMR} \quad\left(500 \mathrm{MHz}, \mathrm{CDCl}_{3}\right): \quad \delta \quad$ (ppm) 3.24 $\left(-\mathrm{N}-\mathrm{CH}_{2}-\mathrm{CH}_{2-}, \quad\right.$ t, $\left.\quad 2 \mathrm{H}\right), \quad 2.94 \quad\left(-\mathrm{N}-\mathrm{CH}_{3}, \quad \mathrm{~s}, \quad 3 \mathrm{H}\right), \quad 2.32$ $\left(\mathrm{CH}_{2}-\mathrm{CH}_{2}-\mathrm{N}-, \quad\right.$ t, $\left.2 \mathrm{H}\right), \quad 2.21 \quad\left(-\mathrm{N}-\mathrm{CH}_{3^{-}}, \quad \mathrm{s}, \quad 6 \mathrm{H}\right), \quad 1.71$ $\left(\mathrm{CH}_{2}-\mathrm{CH}_{2}-\mathrm{CH}_{2-}, \mathrm{m}, 2 \mathrm{H}\right)$. FTIR (KBr pellet, $\left.\mathrm{cm}^{-1}\right): 2941$ (s, alkyl-H), 2815 (s, alkyl-H), 2765 (s, alkyl-H), 2125 (s, N=C).

\subsubsection{1-(3-aminopropyl)-4-methylpiperazinemethyl Carbodiimide}

1-(3-aminopropyl)-4-methyl piperazine amine (1.0 g, 6.358 $\mathrm{mmol})$ in DCM reacted with methyl isothiocyanate $(0.93 \mathrm{~g}$, $6.358 \mathrm{mmol})$ at $0^{\circ} \mathrm{C}$. After $24 \mathrm{hrs}$ of stirring $\mathrm{HgO}(2.82 \mathrm{~g}, 13.02$ $\mathrm{mmol})$ and $\mathrm{MgSO}_{4}(2.35 \mathrm{~g}, 21.7 \mathrm{mmol})$ were added to the flasks respectively. The monomer formed isolated by column chromatography, silica as the stationary phase, and DCM as the eluent. The carbodiimide was a colorless liquid, yield (40\%). 1H NMR (500 MHz, $\left.\mathrm{CDCl}_{3}\right): \delta$ (ppm) $2.94\left(-\mathrm{N}-\mathrm{CH}_{3}, \mathrm{~s}\right.$, $3 \mathrm{H}), 3.24\left(-\mathrm{N}-\mathrm{CH}_{2}-\mathrm{N}-\right.$, t, $\left.4 \mathrm{H}\right), 2.27\left(-\mathrm{N}-\mathrm{CH}_{3}, \mathrm{~s}, 3 \mathrm{H}\right), 2.40$ $\left(-\mathrm{N}-\mathrm{CH}_{2}-\mathrm{CH}_{2-}, \mathrm{t}, 4 \mathrm{H}\right), 1.74\left(\mathrm{CH}_{2}-\mathrm{CH}_{2}-\mathrm{CH}_{2}-\right.$, m, 2H). FTIR (KBr pellet, cm-1): 2957 (s, alkyl-H), 2837 (s, alkyl-H), 2820 (s, alkyl-H), 2123 (s, N=C).

\subsubsection{Synthesis of Polymers}

$1.00 \mathrm{~g}$ of each monomer was dissolved in $0.4 \mathrm{ml}$ of anhydrous chloroform and reacted with (1: 200) $S$-Binol Ti (IV) isopropoxy catalyst. The reaction performed for $12 \mathrm{~h}$ until the reaction mixture gelled and thickened.

The polymerization performed in the glove box at $25^{\circ} \mathrm{C}$ until otherwise noted. After completion of the reaction, the vial was taken out from the glove box and dissolved the polymer sample with chloroform, and precipitate it using hexane.

i. Poly[1-(3-aminopropyl)-4-methylpiperazinemethyl carbodiimide] - Poly-1-S

$S$-Binol Ti (IV) catalyst $(0.02 \mathrm{~g}, 0.05 \mathrm{mmol})$ used to react with 1-(3-aminopropyl)-4-methylpiperazinemethyl carbodiimide (1.87 g, $9.52 \mathrm{mmol})$, (Yield 48\%), Mn=2375 Da, $\mathrm{Mw}=9856 \mathrm{Da} .1 \mathrm{H} \mathrm{NMR}\left(500 \mathrm{MHz}, \mathrm{CDCl}_{3}\right): \delta(\mathrm{ppm}) 3.20(\mathrm{~b}$, $\left.-\mathrm{N}-\mathrm{CH}_{2}\right), 3.10\left(\mathrm{~s},-\mathrm{N}-\mathrm{CH}_{3}\right), 2.35\left(\mathrm{~b},-\mathrm{CH}_{2}-\mathrm{N}\right), 2.18\left(\mathrm{~s},-\mathrm{N}-\mathrm{CH}_{3}\right)$, 1.67 (b, methane-H). $25^{\circ} \mathrm{C}[\alpha]_{435 \mathrm{~nm}}=-14.5^{\circ}$ (chloroform). 
ii. Poly[3-(3-Dimethylaminopropyl)-1-methylcarbodiimide] - Poly-2-S

3-(3-Dimethylaminopropyl)-1-methylcarbodiimide (1.22 g, $8.65 \mathrm{mmol}$ ) reacted with $S$-Binol Ti (IV) catalyst (19 mg, 0.04 mmol). (Yield 72\%), Mn=3941 Da, Mw=28 KDa. 1H NMR $\left(500 \mathrm{MHz}, \mathrm{CDCl}_{3}\right): \delta(\mathrm{ppm}) 3.20\left(\mathrm{~b}, \mathrm{CH}_{2}-\mathrm{N}\right), 3.03\left(\mathrm{~s},-\mathrm{N}-\mathrm{CH}_{3}\right)$, $2.35\left(\mathrm{~b},-\mathrm{N}-\mathrm{CH}_{2}\right), 1.74(\mathrm{~b}$, methane $-\mathrm{H}) .25^{\circ} \mathrm{C}[\alpha]_{435 \mathrm{~nm}}=-29.4^{\circ}$ (chloroform).

\subsubsection{Physical Micelle Stability}

CMC values calculated by fluorescence spectroscopy using pyrene as a probe. the aqueous stock polymer micelle solution $(2 \mathrm{mg} / \mathrm{mL})$ was prepared. It was further diluted into different polymer concentration range between $(1 \mathrm{mg}-0.1 \mu \mathrm{g} / \mathrm{mL})$. the constant amount $(0.5 \mu \mathrm{M})$ of pyrene was added to the different concentrations of polymer solutions at room temperature. The emission spectrum was obtained between $350-450 \mathrm{~nm}$ and the excitation wavelength would be $333 \mathrm{~nm}$. The bandwidth of the spectrum slit was $5 \mathrm{~nm}$. The intensity ratio of pyrene (384 $\mathrm{nm}: 373 \mathrm{~nm}$ ) plotted against $\log \mathrm{C}$ (logarithm value of micelle concentration) and the Critical Micelle Concentration (CMC) can be calculated from the intersection point of the curve. [11-13]

\subsubsection{Particle Size of the Micelles}

Dynamic Light Scattering (DLS) measurements have been taken to analyze the size of the micelle (Malvern Instruments, Zetasizer Nano ZS). The polymer samples were dissolved in deionized water and phosphate buffer solutions $(\mathrm{pH}=7.3,6.0$, 4.6) at room temperature. Each polymer solution concentration was $1 \mathrm{mg} / \mathrm{mL}$. A few drops of the polymer solutions were transferred onto a 300 mesh $\mathrm{Cu}$ grid of lacey carbon and dried at room temperature. Subsequently, the aggregated polymer morphologies were probed using a JEOL $2100 \mathrm{~F} 120 \mathrm{keV}$ transmission electron microscope (TEM). [15-19]

\subsubsection{Encapsulation of Nile Red}

Both polymer and the Nile red dissolved in a $50 \mu \mathrm{L}$ amount of THF and stirred together. The solution mixture was slowly added to the vigorously stirring deionized water. The solution was filtered through a $0.45 \mu \mathrm{m}$ filter and filtrate transferred to the dialysis bag ( $3500 \mathrm{MW}, 5$ inches), which was then sealed and dialyze with deionized water for $24 \mathrm{hrs}$ to remove the organic solvent and uncaptured Nile red molecules. The fluorescent spectroscopy was used to measure the encapsulated Nile red molecules. The emission wavelength of Nile red at $620 \mathrm{~nm}$ and excitation band at $570 \mathrm{~nm}$. The Nile red concentration was calculated by the standard preestablished curve. Nile red loading capacity (LC) and loading efficiency (LE) is calculated by the given equation below. [14, 20-22]

$$
\text { LC } \%=\frac{\text { Weight of the loaded Nile red }}{\text { Weight of the polymer }} \times 100 \%
$$

$$
\mathrm{LE} \%=\frac{\text { Weight of the loaded Nile red }}{\text { Weight of the feed Nile red }} \times 100 \%
$$

\section{Results and Discussion}

\subsection{Preparation of Amphiphilic Polycarbodiimide Nanoparticles}

Previous research has been carried out to study the properties of polycarbodiimides including liquid crystalline, chiroptical switching, asymmetric solid support to name a few. [23-25] Thus, in this study, we are identifying the self-assemble properties of the carbodiimide polymers and the feasibility to use them as stimuli-responsive nanocarriers.

We are first time reporting amphiphilic polycarbodiimide homopolymers that have $\mathrm{pH}$-dependent self-assembling properties. These polycarbodiimides are relatively easy to synthesize and reproducible. The nitrogen-rich polymer backbone and introduced piperazine and dimethylamine functional groups enhanced the $\mathrm{pH}$-dependent swelling properties of the nanoscale micelles. Most of the previously reported $\mathrm{pH}$-triggered self-assembled polymers like polyethylene glycol, poly ( $N$-isopropyl acrylamide), poly ( $N$-vinylpyrrolidone), polycaprolactone, polyoxazoline, to name a few were block-copolymers. [14, 26-28] Thus, here we are introducing new set of polycarbodiimide homopolymers the poly-1-S and poly-2-S that showed satisfactory drug loading ability and stimuli-responsive property.

Block-co-polymers are consisting of both hydrophobic and hydrophilic segments. The aggregation of these polymers depends upon the solvent in the medium. Self-assemblence of these polymers taken place either in a mixture of solvents or in a specific solvent system. Generally, with the presence of polar solvents like water, hydrophilic chains start to interact with water molecules and the hydrophobic portion of the polymer starts to assemble in a way to form aggregations. However, when the organic solvent is present the hydrophobic segment is dissolved in the medium, and the hydrophilic segment is assembled in a way to form micelles where the hydrophilic portion sticks inside. Thus, these block-copolymers dissolved in both polar and nonpolar solvent systems to form micelles. The amphiphilic homopolymers generally consist of hydrophilic and hydrophobic side groups in each repeat unit of the polymer that can self-assemble itself to form micelles in the presence of both organic and polar solvent systems as well as the mixture of solvents.

In this work, we designed carbodiimide polymers, each repeat unit consists of both hydrophobic and hydrophilic functional groups. i.e., polar side groups like piperazine and dimethylamine attached to the imine nitrogen and methyl group attached to the amine nitrogen as shown in Figure 2 to make amphiphilic polycarbodiimides. The polymerizations were carried out in the presence of $S$-Binol Ti isopropoxide catalyst with corresponding amphiphilic monomer as shown in Figure 2. 
<smiles>CN=C=NCCCN1CCN(C)CC1</smiles>

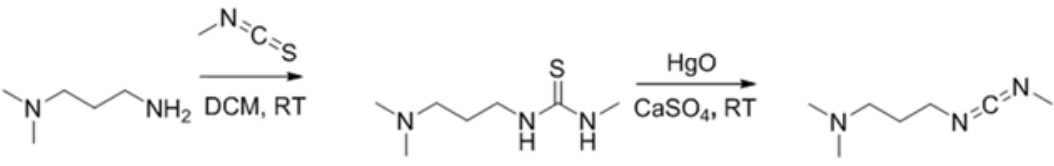<smiles>CN=C=NCCCN1CCN(C)CC1</smiles>

Figure 2. Synthesis of amphiphilic polycarbodiimides.

All these carbodiimide polymers are readily soluble in both polar and nonpolar solvents including water, methanol, dioxane, dimethylformamide, tetrahydrofuran, chloroform, dichloromethane, to name a few. Thus, these polymers can be used to study their self-assembly behavior.

The self-assembling properties of these polymers were investigated by Dynamic Light Scattering (DLS) results. When amphiphilic polycarbodiimide is mixed with water, hydrophobic functional groups congregate to form aggregates. Thus, in general, hydrophobic moieties stick inside in a way that hydrophilic parts resemble outside micelle core due to hydrogen bonding or polar interaction forming between water
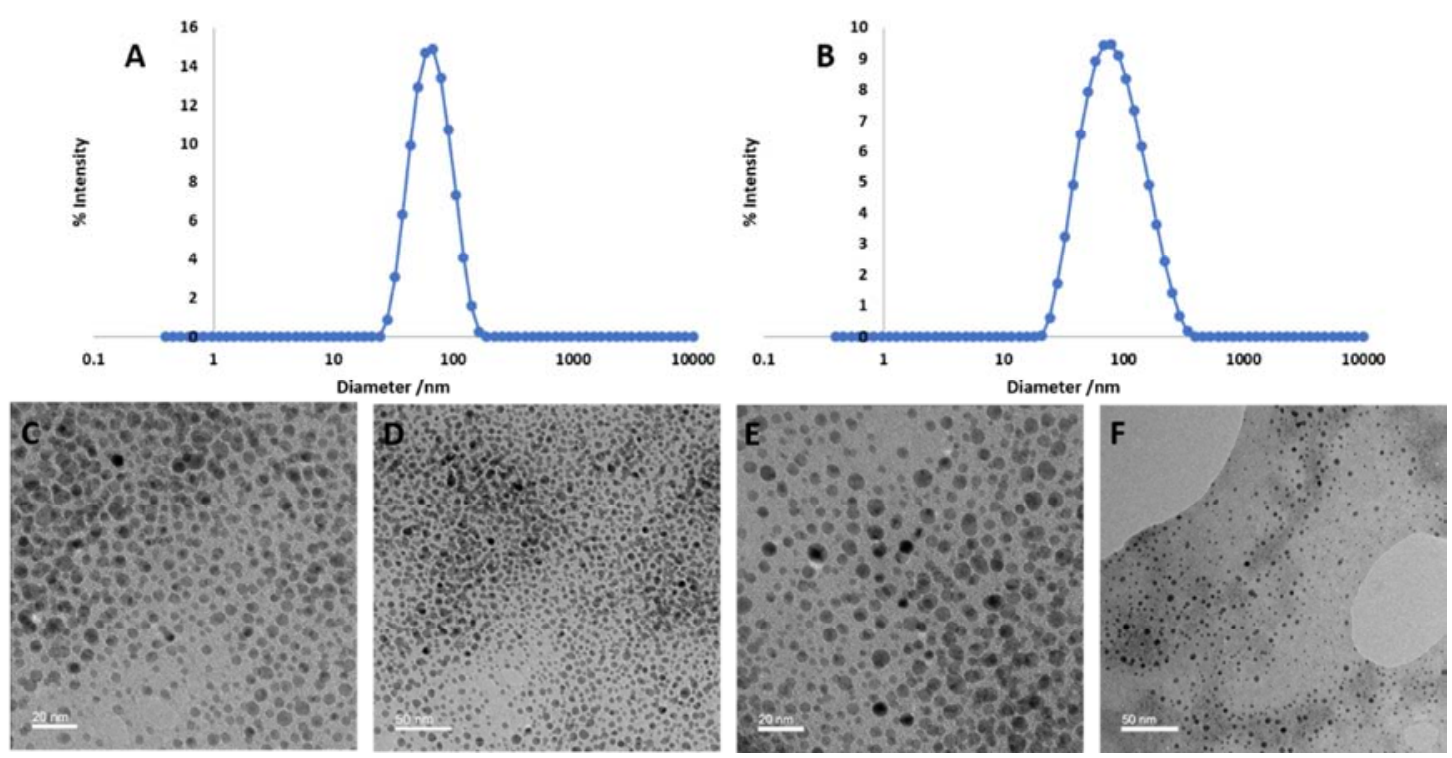

Figure 3. Size distribution of Poly-1-S (A) and Poly-2-S (B) in deionized water. TEM images of Poly-1-S (C, D) and Poly-2-S (E, F) cast from deionized water.

molecules and piperazine and dimethylamine functional groups. The size and shape of the micelles can differ from spherical, cylinders, vesical, and lamella shapes.

The size distribution of the aggregated polymer particles in an aqueous medium can determine through DLS results (Figure 3). The results indicated that these carbodiimide polymers self-assembled in water to form micelles. According to the DLS data, the micelles can consider as nanoparticles, because the size of the polymeric particles distributed in the range of 10-100 $\mathrm{nm}$. The size distribution of the micelle particles of both polymers (Poly-1-S and Poly-2-S) are similar.

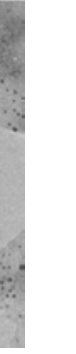


The shape of the polymer particles was studied by transmission electron microscopy (TEM). Both polycarbodiimide polymers (Poly-1-S and Poly-2-S) dissolved in water to obtain an aqueous polymer solution $(1 \mathrm{mg} / \mathrm{mL})$. The morphology of the polydisperse particles in Figure 3 indicates that the polymers self-assembled in water to form sphere shape micelles. Thus, these polycarbodiimides can be used as nanocarrier materials.

The critical micelle concentration (CMC) is an important measurement to identify the stability of the micelle in different concentrations. the critical micelle concentration showed that both polymers successfully form stable micelles. Pyrene was used to measure the $\mathrm{CMC}$ of the polymers, which was determined as $0.308 \mathrm{mg} / \mathrm{ml}$ for poly- $1-S$ and $0.004 \mathrm{mg} / \mathrm{ml}$ for poly-2-S. the lower CMC values indicate the stability of the micelles, as these nanoparticles maintain their morphology without breaking them apart upon dilution and systemic delivery, which would be excellent candidates for in vivo nanocarrier applications.

\subsection{Nile Red Loading Ability}

Micellization of the polycarbodiimides upon dissolving in water was identified by CMC and DLS results. The loading capacity and efficiency of micelles can be determined by using Nile red. Nile red is a dye which is dissolved only in organic solvents and fluorescent active. When Nile red is encapsulated into the micelle hydrophobic core, it was capable to produce fluorescence. Thus, Nile red can be used as an indicator to measure micellization as well as the molecular loading capacity of the micelles.

Micelle Nile red loading capacity and loading efficiency of the homopolymers were measured by fluorescence spectroscopy. We were able to obtain relatively low loading efficiency as reported in Table 1, however by making block-copolymers, we might be able to increase the loading efficiency. Also incorporating long-chain branched alkyl groups and more hydrophilic pendant groups enhances the loading efficiency of the homopolymers. Further, we can use Ni or Ti catalyst to make block-copolymers which initiate living polymerization.

Table 1. Characterization of polycarbodiimide micelles.

\begin{tabular}{lllll}
\hline Micelles & $\begin{array}{l}\text { DLC (wt.\%) (Nile red loading } \\
\text { capacity) }\end{array}$ & $\begin{array}{l}\text { DLE\% (Nile red loading } \\
\text { efficiency) }\end{array}$ & $\begin{array}{l}\text { Z-Average (d.nm) } \\
\text { (Zeta-Average) }\end{array}$ & $\begin{array}{l}\text { PDI (Polydispercity index) } \\
\text { Poly-1-S }\end{array} 0^{\text {Poly-2-S }}$ \\
\hline
\end{tabular}

\section{3. pH Dependent Micelle Stability}

Amphiphilicity of the polycarbodiimides depends upon the hydrophobic and hydrophilic pendant groups. The invented piperazine and dimethylamine functional groups and nitrogen-rich polymer backbone made carbodiimide polymers known as basic polymers. These basic polymers are a good source for $\mathrm{pH}$-triggered applications. Herein we report the micelle behavior of these self-assemble polycarbodiimides by changing the $\mathrm{pH}$.

The $\mathrm{pH}$-dependent micelle behavior is observed by DLS as a function of $\mathrm{pH}$ and time. The polymeric material was dissolved in different phosphate buffer solutions $(\mathrm{pH} 7.3,6.0)$

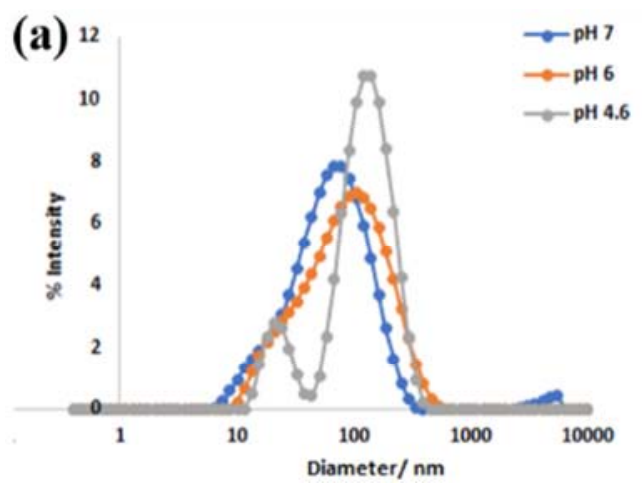

Figure 4. Micelle behavior in different buffer solutions; particle distribution of Poly-1-S (a), and Poly-2-S (b).

The particle size of the alkaline polycarbodiimide treated with neutral buffer solution retain constant, but with the time slight collapsed can be observed after $3 \mathrm{~h}$. however, polymers

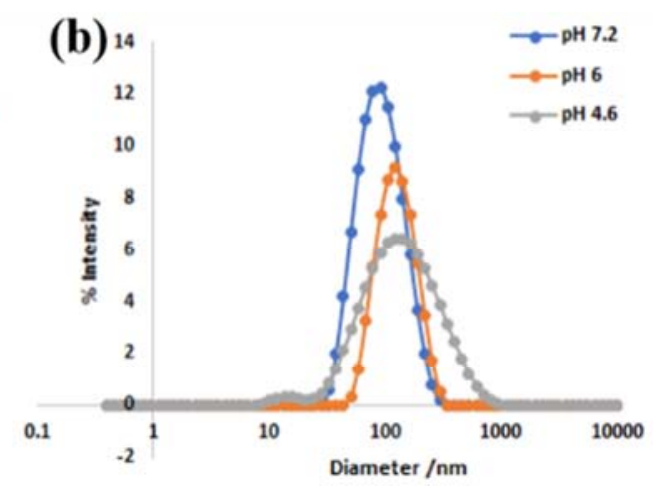

and an acetic acid buffer solution ( $\mathrm{pH} 4.6)$. The architecture of the polycarbodiimide we prepared was basic. Because the piperazine and dimethyl amines are basic functional groups, also the polymer consist of a nitrogen-rich backbone, which defines as a basic polymer. Thus, as shown in Figure 4 , the polymeric micelles start to change size as a function of $\mathrm{pH}$. The size of the micelles gradually increases with decreasing $\mathrm{pH}$, because the polymer backbone, as well as basic pendant groups, starts to protonate in an acid environment which increases the hydrophilicity of the micelles. i.e., protonated micelles attract more water molecules in aqueous media, which swell the micelles. Therefore, the particle size of the polymer treated with $\mathrm{pH} 4.6$ buffer is higher than $\mathrm{pH} 6.0$.

treated with $\mathrm{pH} 4.6$ and $\mathrm{pH} 6.0$ buffer, the degree of swelling increases after $2 \mathrm{~h}$ and partially destroyed the micelles, which form cracked particles corresponding to the peaks around 10 
$\mathrm{nm}$ and $1000 \mathrm{~nm}$ as shown in Figure 5.
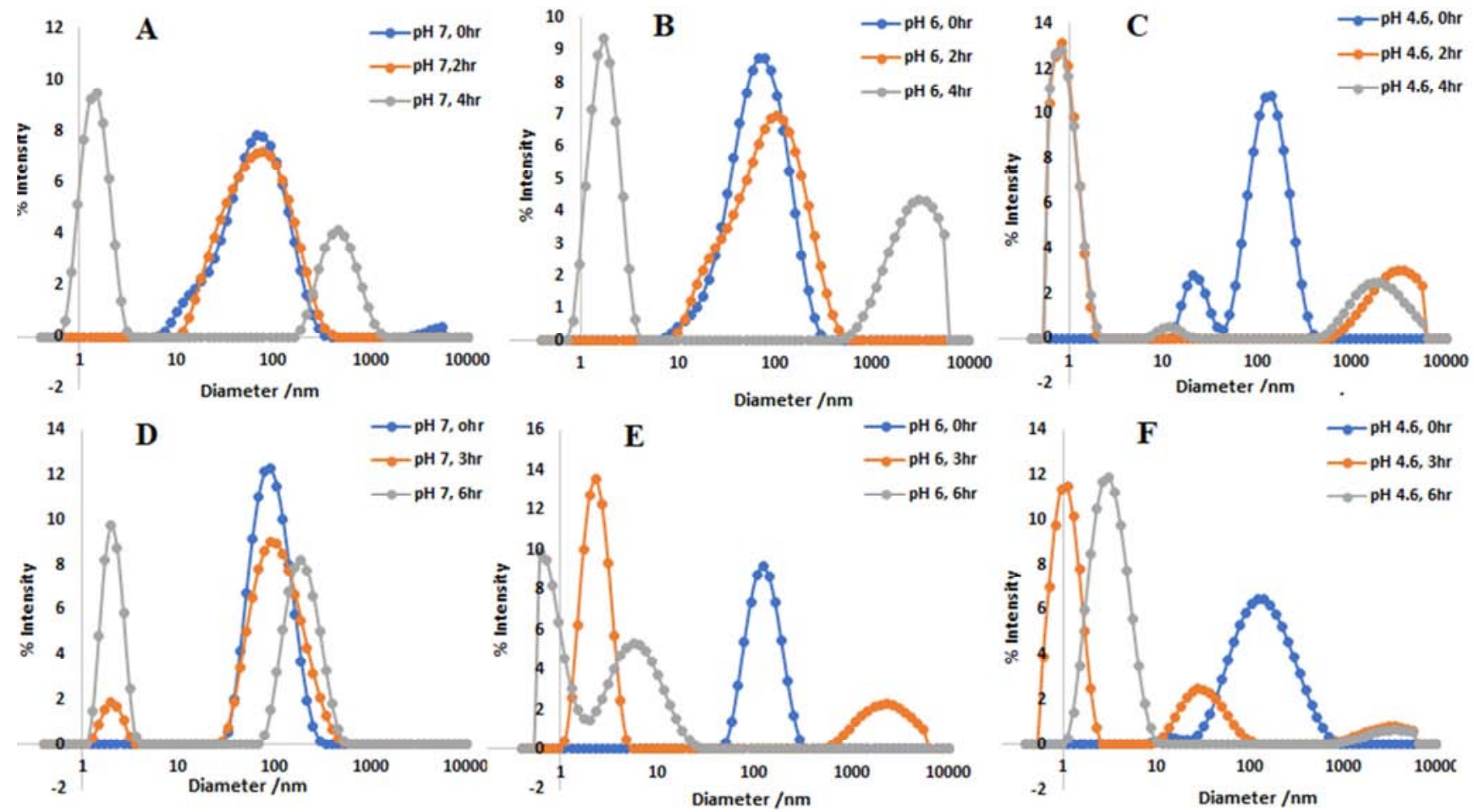

Figure 5. Micelle behavior treated in different buffer solutions depending on time at $37^{\circ} \mathrm{C}$. Poly-1-S; $\mathrm{pH} 7.3(\mathrm{~A}), \mathrm{pH} 6.0$ (B), and pH 4.6 (C). The micelles stability of Poly-2-S; $p H 7.3(D), p H 6.0(E)$, and $p H 4.6(F)$. The peaks showing aggregation size change as a function of time in different buffer solutions (pH: $-7.3,6.0,4.6)$.

After $4 \mathrm{~h}$ retain in the acidic medium, the polymeric micelles swell further to form larger aggregates, that represent by peaks that appeared around $3000 \mathrm{~nm}$. The reduced size peaks appeared at 5-10 nm correspond to the extricated polymeric chains. Thus, after $6 \mathrm{~h}$, the polycarbodiimides treated in acidic buffer solution were protonated and dissociated micelles into tangled polymer fragments. As shown in Figure 5, the self-assembled polycarbodiimides are stable in neutral conditions (pH: -7.3 at $37^{\circ} \mathrm{C}$ ). However, in an acidic medium, the polymer starts to protonate and destroy the aggregate; thus, these polymers are suitable for cancer cell-targeted drug delivery applications.

\section{Conclusion}

In summary, we have successfully invented novel polycarbodiimides materials with water-soluble and $\mathrm{pH}$-sensitive properties. The architecture of the polymer was modified by incorporating polar functional groups which enhanced the hydrophilicity and $\mathrm{pH}$ sensitivity. Interestingly these homopolymers self-assembled in an aqueous medium. The loading capacity of the micelles was $\sim 0.8 \%$ and it can further improve by modified the structure with hydrophobic alkyl chains to the polymer backbone. The polymer aggregates were stable in the normal physiological environment $\left(\mathrm{pH} 7.4,37^{\circ} \mathrm{C}\right)$ and they are started to disassemble in acidic conditions. Therefore, these polymers can be used as a nanocarrier, where perform stability in prolong circulation time, dissociates, and release molecules or drugs in a mildly acidic environment. Thus, the polymeric vesicles could be a potential candidate for cancer therapy.

\section{Acknowledgements}

Kindly acknowledged Dr. Kenneth Balkus and Alexander Brown for helping me to collect TEM images. Also, I am thankful to the Department of Chemistry and Biochemistry, the University of Texas at Dallas for the funding.

\section{References}

[1] Kocak, G.; Tuncer, C.; Butun, V. pH-Responsive polymers. Polym. Chem. 2017, 8, 144-176.

[2] James, H. P.; John, R.; Alex, A.; Anoop, K. R. Smart polymers for the controlled delivery of drugs - a concise overview. Acta Pharmaceutica Sinica B 2014, 4, 120-127.

[3] Schmaljohann, D. Thermo and pH-responsive polymers in drug delivery. Advanced drug delivery reviews 2006, 58, 1655-1670.

[4] Zhang, J.; Liu, K.; Mullen, K.; Yin, M. Self-assemblies of amphiphilic homopolymer: synthesis, morphology studies and biomedical applications. Chem. Commun. 2015, 51, 11541-11555.

[5] Zhou, Y.; Liu, B.; Wang, X. Self-assembly of homopolymers through strong dipole-dipole interaction in their aqueous solutions. Polymer 2016, 97, 1-10. 
[6] Sun, T.; Yang, X.; Zhu, C.; Zhao, N.; Dong, H.; Xu, J. Self-assembly of homopolymer of PAA-NH ${ }_{4}$. Chin. Chem. Lett. 2018, 1-4.

[7] Zhu, Y.; Yang, Q.; Tong, C.; Li, M.; Yu, X. The vesicle formation in a binary amphiphilic diblock copolymer/homopolymer solution. Polymer 2010, 51, 702708 .

[8] Hua, D.; Tang, J.; Jiang, J.; Zhu, X. Synthesis of phenylphosphinic acid-containing amphiphilic homopolymers by reversible addition-fragmentation transfer (RAFT) polymerization and its aggregation in water. Polymer 2009, 50, 5701-5707.

[9] Sedlak, M.; A novel approach to controlled self-assembly of $\mathrm{pH}$-responsive thermosensitive homopolymer polyelectrolytes into stable nanoparticles. Advances in Colloid and Interface Science 2016, 232, 57-69.

[10] Yia, Y.; Lin, G.; Chen, S.; Liu, J.; Zhang, H.; Mi, P. Polyester micelles for drug delivery and cancer theranostics: Current achievements, progresses and future perspectives. Materials Science \& Engineering C 2018, 83, 218-232.

[11] Liu, X.; Thomas, K. J. Study of surface properties of clay laponite using pyrene as a photophysical probe molecule. Langmuir 1991, 7, 2808-2816.

[12] Chin, Y.; Aiken, G. R.; Danielsen, K. M. Binding of pyrene to aquatic and commercial humic substances: the role of molecular weight and aromaticity. Environ. Sci. echnol. 1997, $31,1630-1635$.

[13] Dominguez, A.; Fernandez, A.; Gonzalez, N.; Iglesias, E.; Montenegro, L. Determination of critical micelle concentration of some surfactants by three techniques. Journal of Chemical Education 1997, 74, 1227-1231.

[14] Cheng, Y.; Hao, J.; Lee, L. A.; Biewer, M. C.; Wang, Q.; Stefan, M. C. Thermally controlled release of anticancer drug from self-assembled $\gamma$-substituted amphiphilic poly( $\varepsilon$-caprolactone) Micellar nanoparticles. Biomacromolecules 2012, 13, 2163-2173.

[15] Qiu, L.; Zhu, M.; Gong, K.; Peng, H.; Ge. L.; Zhao, L.; Chen, J. $\mathrm{pH}$-triggered degradable polymeric micelles for targeted anti-tumor drug delivery. Material Science and Engineering $C$ 2017, 78, 912-922.

[16] Jafarzadeh-Holagh, Samira.; Hashemi-Najafabadi, S.; Shaki, H.; Vasheghani-Farahani, E. Self-Assembled and $\mathrm{pH}$ sensitive mixed micelles as an intracellular doxorubicin delivery system. Journal of Colloid and Interface Science 2018, 523, 179-190.

[17] Xiao, L.; Huang, L.; Moingeon, F.; Gauthier, M.; Yang, G. pH-Responsive poly (ethylene glycol)-block-polylactide micelles for tumor-targeted drug delivery. Biomacromolecules 2017, 18, 2711-2722.

[18] Huang, Y.; Tang, Z.; Zhang, X.; Yu, H.; Sun, H.; Pang, X.; Chen, $\mathrm{X}$. pH-Triggered charge-reversal polypeptide nanoparticles for cisplatin delivery: preparation and in vitro evaluation. Biomacromolecules 2013, 14, 2023-2032.

[19] Wang, Y.; Nie, J.; Chang, B.; Sun, Y.; Yang, W. Poly(vinylcaprolactam)-based biodegradable multiresponsive microgels for drug delivery. Biomacromolecules 2013, 14, 3034-3046.

[20] Li, Y.; Bui, Q. N.; Duy, T. M.; Yang, H. Y.; Lee, D. S. One-step preparation of $\mathrm{pH}$-responsive polymeric nanogels as intelligent drug delivery systems fort tumor therapy. Biomacromolecules 2018, 19, 2062-2070.

[21] Huang, F.; Cheng, R.; Meng, F.; Deng, C.; Zhong, Z. Micelles based on acid degradable poly (acetal urethane): preparation, $\mathrm{pH}$-sensitivity, and triggered intracellular drug release. Biomacromolecules 2015, 16, 2228-2236.

[22] Yu, Y.; Chen, C.; Law, W.; Weinheimer, E.; Sengupta, S.; Prasad, P. N.; Cheng, C. Polylactide-graft-doxorubicin nanoparticles with precisely controlled drug loading for pH-triggered drug delivery. Biomacromolecules 2014, 15, 524-532.

[23] Tang, H. Z.; Boyle, P. D.; Novak, B. M. Chiroptical Switching Polyguanidine Synthesized by Helix-Sense-Selective Polymerization Using [(R)-3,3'-Dibromo-2,2'-binaphthoxy] (di-tert-butoxy) titanium (IV) Catalyst. J. Am. Chem. Soc. 2005 , 127, 2136-2142.

[24] Reuther, J. F.; Novak, B. M. Evidence of Entropy-Driven Bistability through ${ }^{15} \mathrm{~N}$ NMR Analysis of a Temperature and Solvent Induced, Chiroptical Switching Polycarbodiimide. $J$. Am. Chem. Soc. 2013, 135, 19292-19303.

[25] Kennemur, J. G.; Novak, B. M. Advances in polycarbodiimide chemistry. Polymer 2011, 52, 1693-1710.

[26] Sideratous, Z.; Agathokleous, M.; Theodossious, T. A.; Tsiourvas, D. functionalized hyperbranched polyethylenimines as thermosensitive drug delivery nanocarriers with controlled transition temperature. Biomacromolecules 2018, 19, 315-328.

[27] Jin, Y.; Song, L.; Su, Y.; Zhu, L.; Pang, Y.; Qiu, F.; Tong, G.; Yan, D.; Zhu, B.; Zhu, X. oxime linkage: a robust tool for the design of $\mathrm{pH}$-sensitive polymeric drug carriers. Biomacromolecules 2011, 12, 3460-3468.

[28] Chen, W.; Zhou, S.; Ge, L.; Wu, W.; Jiang, X. translatable high drug loading drug delivery systems based on biocompatible polymer nanocarriers. Biomacromolecules 2018, 19, 1732-1745. 